\title{
Radiographic abnormalities in eyes with retinoblastoma and other disorders
}

\author{
GORDON K. KLINTWORTH \\ From the Departments of Pathology and Ophthalmology, Duke University Medical Center, Durham, \\ North Carolina, USA
}

SUMMARY The importance of radiographic examinations of pathological ocular material is stressed. Characteristic ocular radiodensities are observed with retinoblastomas, calcified cataracts, senile scleral plaques, intraocular ossification, and a variety of radiodense foreign bodies. Radiographs supplement other techniques for the documentation of ocular abnormalities and in certain instances may be the ideal method. They also permit the precise localisation of radiodense foreign bodies that need to be removed prior to the processing of tissue for microscopic examination. In certain situations valuable data can be obtained by $x$-ray examinations of embedded material. Retinoblastomas have an extremely high incidence of radiodensities with a characteristic appearance. This finding stresses the clinical importance of utilising and developing clinically applicable techniques for the detection of calcification in patients with suspected retinoblastomas.

Roentgenography has entrenched itself as a valuable diagnostic procedure in clinical ophthalmology and in medicine in general. Several texts are specifically devoted to applications of $x$-ray examinations of interest to ophthalmologists (Hartmann and Gilles, 1959; Guillot et al., 1966; Lombardi, 1967). On the other hand scant attention has been paid to radiographic examinations of excised pathological ocular tissues despite the fact that such investigations offer even more opportunity for evaluation as the limitations imposed by the bony orbit are absent. This report reviews experience with radiographic examinations on approximately 200 selected eyes.

\section{Materials and methods}

Since $1969 x$-ray examinations have been performed at Duke University Medical Center on approximately 200 selected eyes with a wide variety of abnormalities. These included phthisis bulbi, uveal melanomas, panophthalmitis, endopthalmitis, cataracts, glaucoma of several types, gunshot wounds, known or suspected foreign bodies, detached retinas, retinoblastomas, Coats's disease, segmental retinal gliosis, retrolental fibroplasia, senile scleral plaques, and microphthalmos. Some eyes had encircling bands for detached retinas or implants of radio-

Address for reprints: Dr Gordon K. Klintworth, Department of Pathology, Duke University Medical Center, Durham, North Carolina, USA opaque materials. Eyes from individuals of various ages with no apparent ocular disease were also studied. Radiographs of all enucleated eyes with retinoblastomas between 1969 and 1977 (24 cases) were obtained. In addition to these cases $x$-ray examinations were performed on portions of other embedded globes (17 Paraplast, 21 paraffin, and 4 celloidin) with retinoblastomas. In all instances the eyes were examined microscopically.

$X$-ray examinations were performed with equipment designed to give high resolution radiographs of small to medium sized objects while operating off an ordinary $110-\mathrm{V}, 50 / 60$ cycle ac line. Most of the roentgenography was carried out with a Picker $110-\mathrm{kV}$ Hotshot portable $x$-ray unit designed primarily for the industrial radiography of light metal. The equipment was mounted on a light metal quadrupod and consisted of a self-contained transformer tubehead equipped with a $0.5-\mathrm{mm}$ thick beryllium window and possessed a $0 \cdot 5-\mathrm{mm}$ focal spot tube for finest detail sensitivity. The total filtration of the $x$-ray beam through the beryllium window ensured a high radiation output within a short time at low $\mathrm{kV}$. Lead shielding was installed between the tube head and an underlying rectangular cabinet $(27.9 \times 26.7 \times 53.3 \mathrm{~cm})$ with $14-\mathrm{mm}$-thick lead walls. The joints between them were lined with 2-mm-thick sheets of lead to provide additional protection. $X$-ray examinations were performed by placing specimens on a shelf in the radiation- 
Figs. 1-3 The calcifications in retinoblastomas are readily visualised in roentgenographs of enucleated eyes
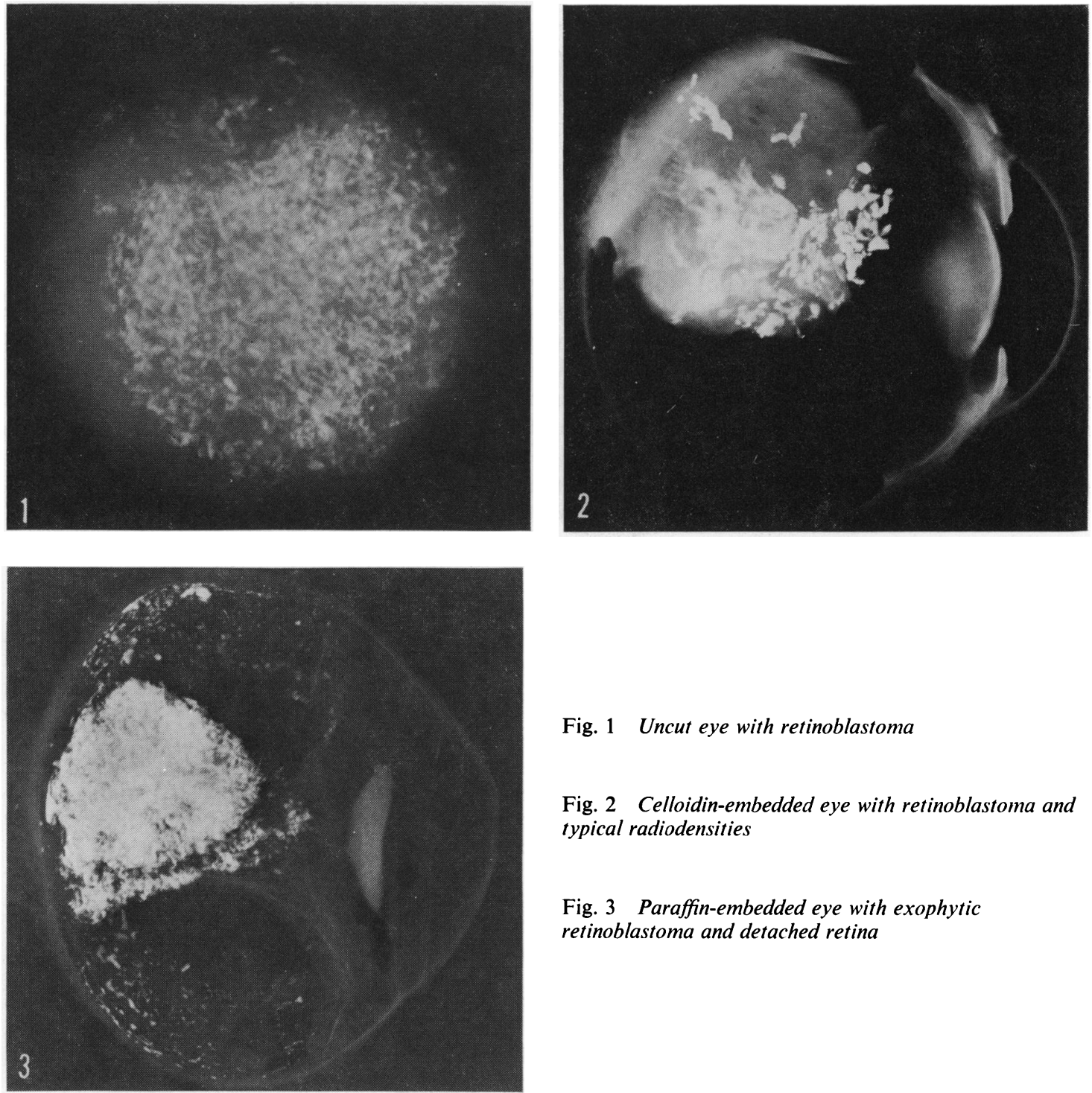

Fig. 1 Uncut eye with retinoblastoma

Fig. 2 Celloidin-embedded eye with retinoblastoma and typical radiodensities

Fig. 3 Paraffin-embedded eye with exophytic retinoblastoma and detached retina

shielded cabinet with the $x$-ray beam directed vertically toward the specimen, which was placed on Kodak industrial $x$-ray film type M. Radiographs of eyes were taken at 20 to $24 \mathrm{kV}$ and $6 \mathrm{~mA}$ with exposure time of 1 minute. After $x$-ray exposure the photographic film was developed for 5 minutes. Recently comparable radiographs have been obtained with a Faxitron $x$-ray System (Model 43805N) (Hewlett Packard, Oregon) with the capability of selecting the optimum $\mathrm{kVp}$ and deter- mining correct exposure time. This equipment has an $0 \cdot 25$-mm-thick beryllium window.

\section{Results}

Numerous distinct radiographic appearances were detected (Figs. 1-14). Of particular importance was the high incidence of characteristic radiodensities in retinoblastomas. These were discrete and relatively uniformly sized (about $1 \mathrm{~mm}$ in diameter) 


\section{Figs. 4-7 Radiographs of eyes}

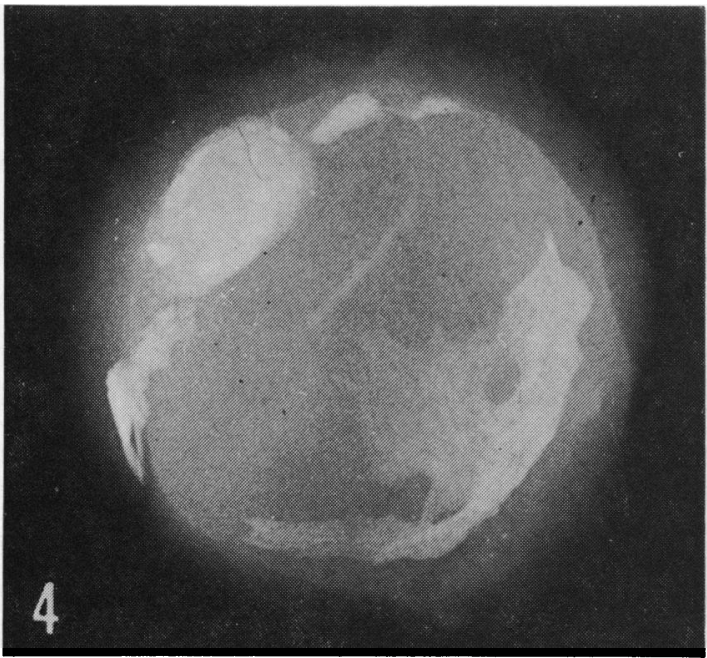

Fig. 4 Central callotte of sectioned phthiscal eye with calcified cataract and bone

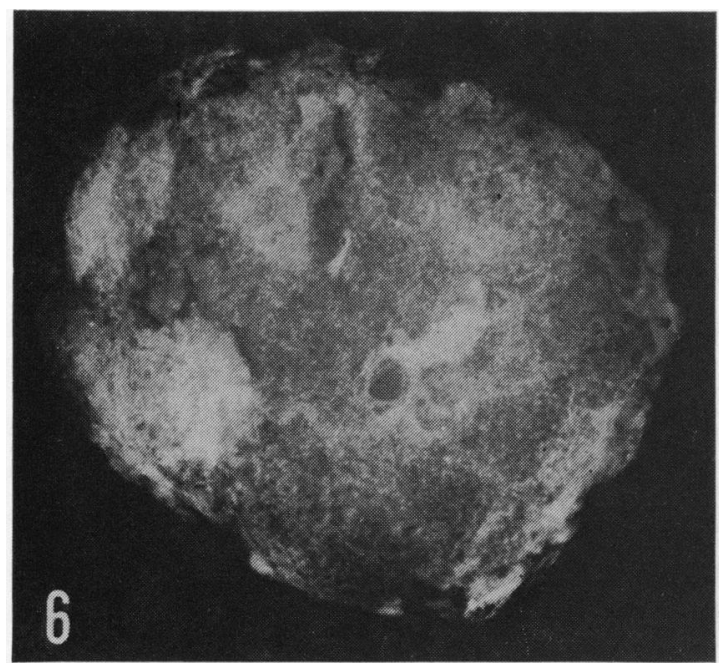

Fig. 6 Extensive ossification in phthisis bulbi

(Figs. 1-3). In parts of some tumours they were abundant, often closely packed and not discernible from each other. Such eyes, nevertheless, still contained smaller discrete opacities (Fig. 3). All 24 non-embedded complete eyes with retinoblastomas had this characteristic radiograph. When only portions of embedded eyes were studied the frequency of positive results varied being $5 / 5(100 \%)$, $16 / 18(89 \%), 12 / 21(57 \%)$ with celloidin, Paraplast, and paraffin embedded tissue respectively. The overall incidence of the typical roentgenographic

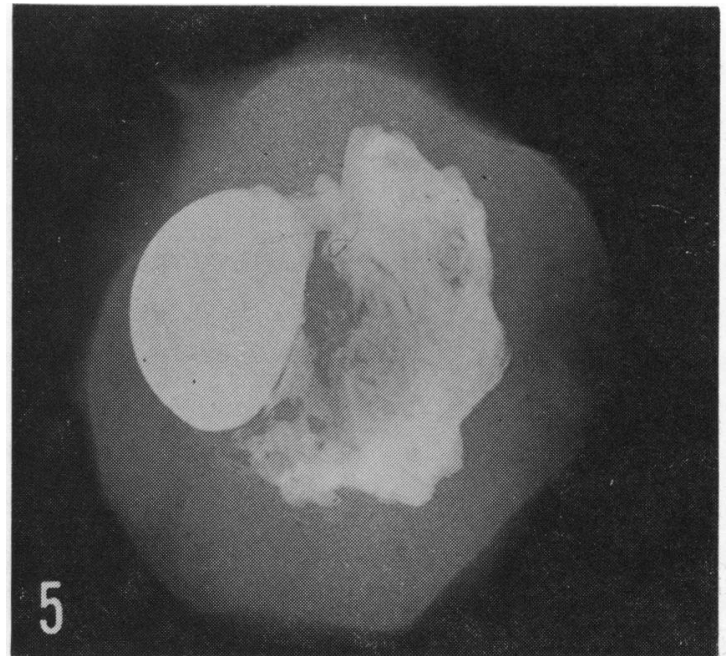

Fig. 5 Calcified cataractous lens and intraocular bone in phthisis bulbi

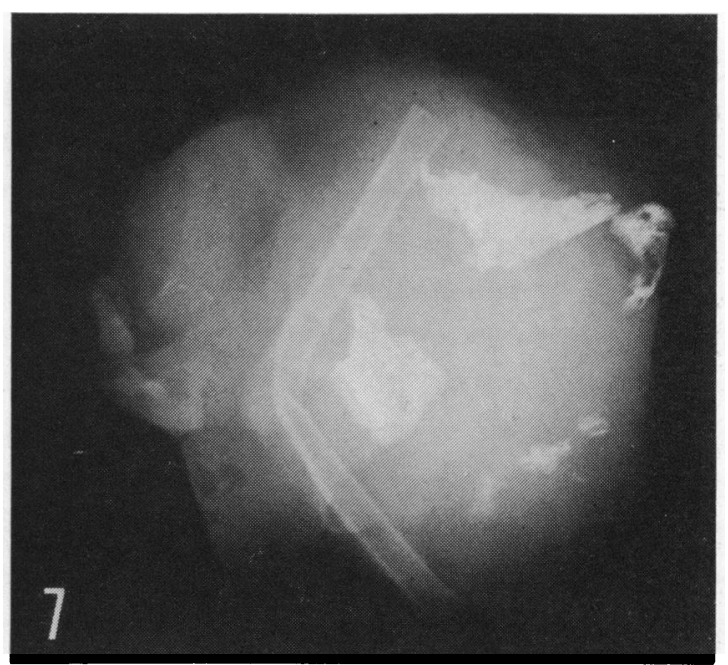

Fig. 7 Intraocular bone and plastic cyclodialysis tube implant

appearance was $84 \%$. All eyes with characteristic radiographic densities contained foci of calcification in tissue sections, but in some instances the calcific deposits were not always present in random sections through the tumour. Radiographs indicated the appropriate region for histological examination which invariably contained calcific deposits. Calcification was not observed in the choroid, optic nerve, or in extraocular extensions of retinoblastomas.

A review of tissue sections of tumours that did 
Figs. 8-11 Radiographs of eyes

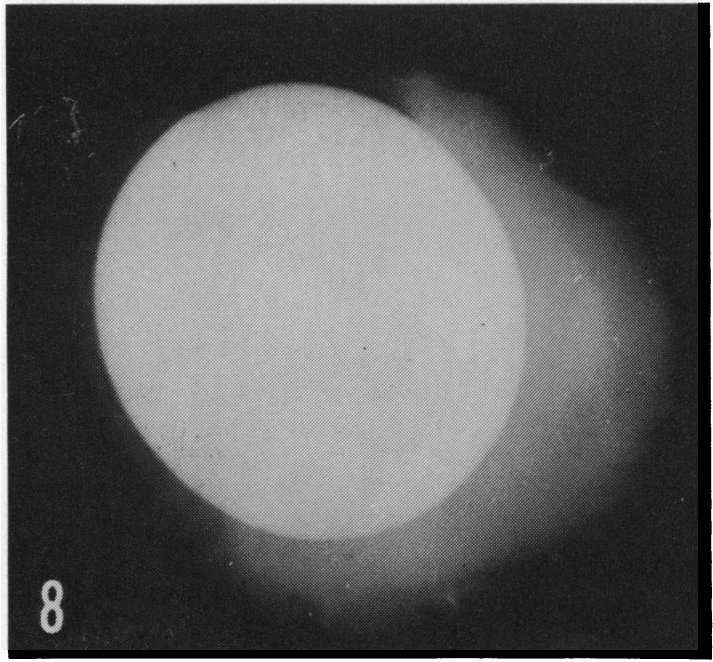

Fig. 8 Siliconised rubber (Silastic implant) within scleral shell of eviscerated eye

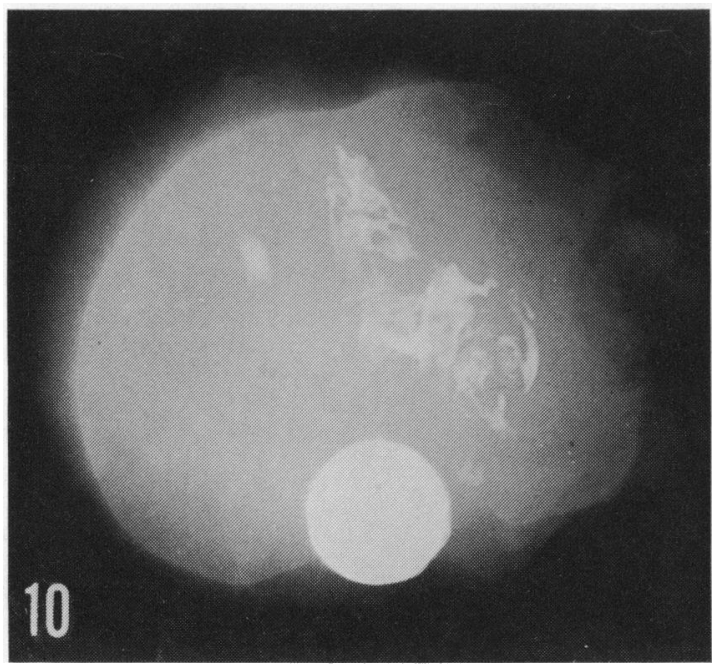

Fig. $10 \quad B B$ pellet and bone in eye of child

not show radiographic abnormalities disclosed that only small fragments of tumour were present in most instances. Some of these retinoblastomas had distinct characteristics. One tumour had destroyed all recognisable ocular components and extended extensively into the periocular tissues, another had much necrosis but no calcification, and 2 others despite massive invasion of the choroid did not manifest zones of necrosis. Additional retinoblastomas were well differentiated with neither necrotic nor calcific foci or small with minimum cellular debris. Three non-radiographically detectable retino-

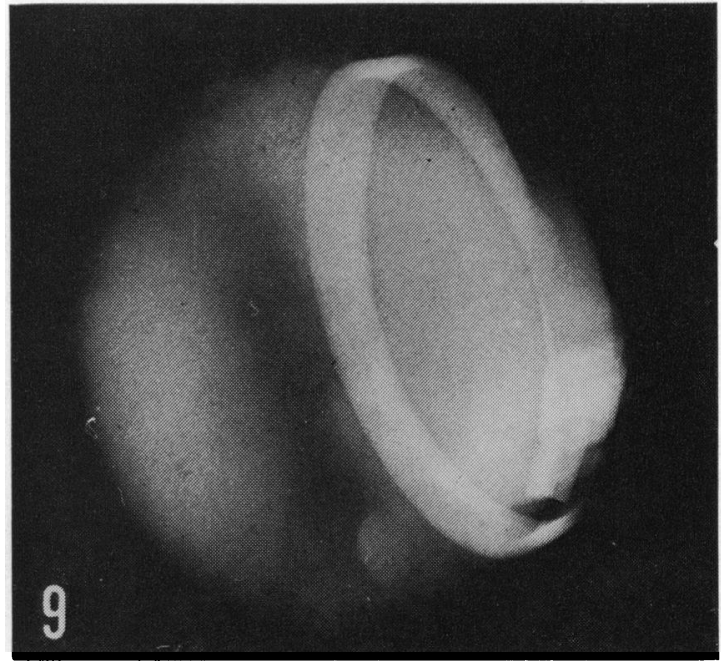

Fig. 9 Encircling band around eye with retinal detachment

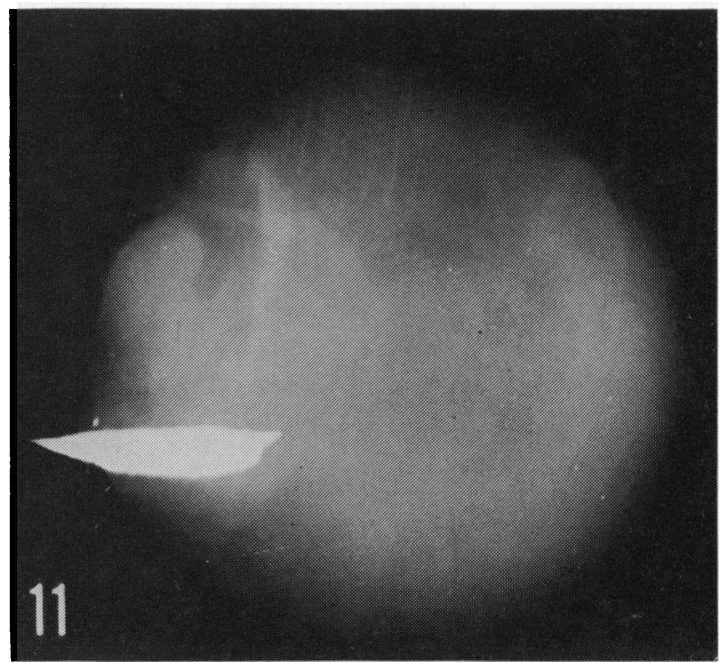

Fig. 11 Metallic foreign body in eye

blastomas contained numerous haematoxyphilic deposits which did not stain with histochemical methods for calcium (alizarin red, von Kossa), or DNA (Feulgen) but stained positively for RNA with methyl green pyronin.

Siliconised rubber (Silastic) and plastic materials used in treatment as in implants of eviscerated eyes, encircling bands for retinal detachment, and cyclodialysis implants dissolved during the normal preparation of tissue sections. They were, however, readily demonstrated by radiographs which formed a permanent record of their presence (Figs. 7-9). 
Figs. 12-14 Radiographs of eyes
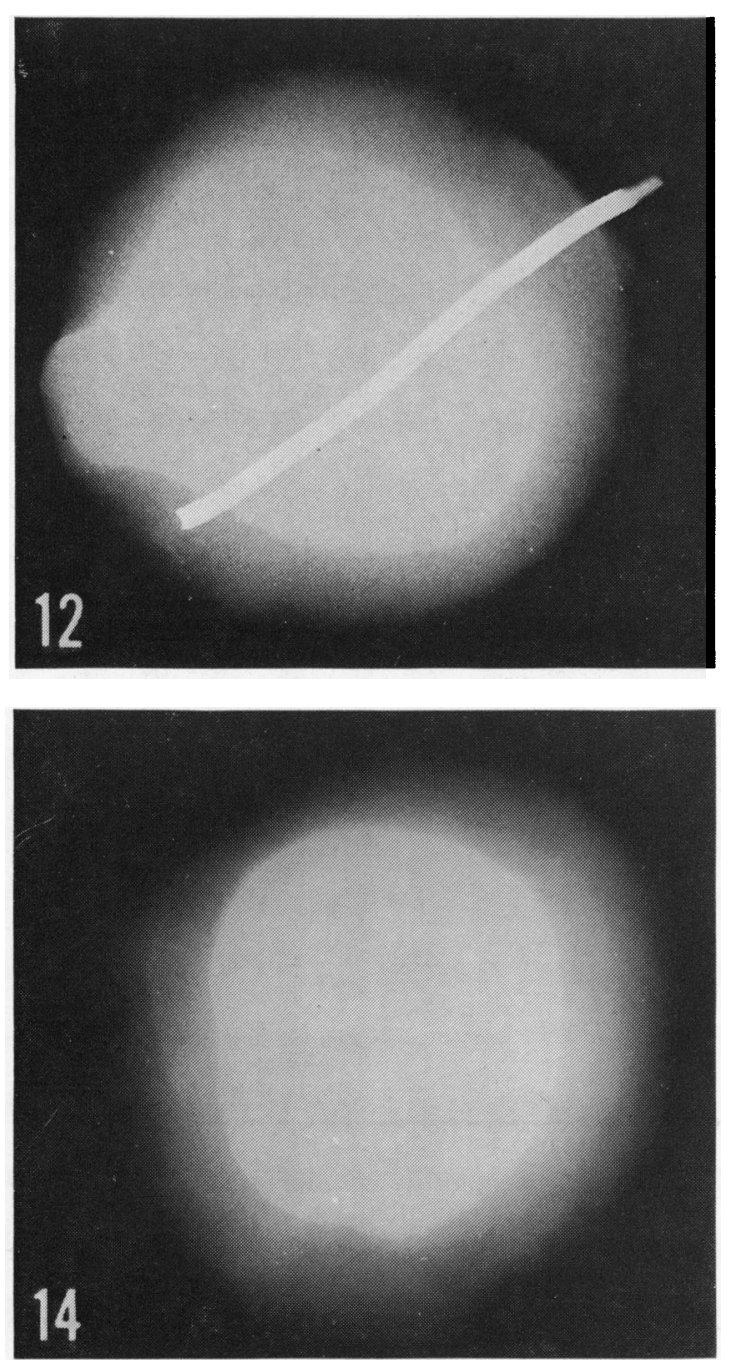

Surprisingly many eyes, especially of elderly individuals, showed an increased radiodensity of the vitreous (Fig. 14). This did not persist after the globes were sectioned and the vitreous merged with the fixative. In some instances it was accompanied by a diffuse but non-radiographically detectable calcification of the sclera or calcific deposits in the ciliary body or ocular lesions. Four cases were associated with glaucoma, but the radiodensity of the vitreous did not reflect increased intraocular pressure. It was almost always found in eyes with normal intraocular pressure, and most glaucomatous eyes did not show this change.

Roentgenographic abnormalities were conspicuously absent in certain ocular disorders, including

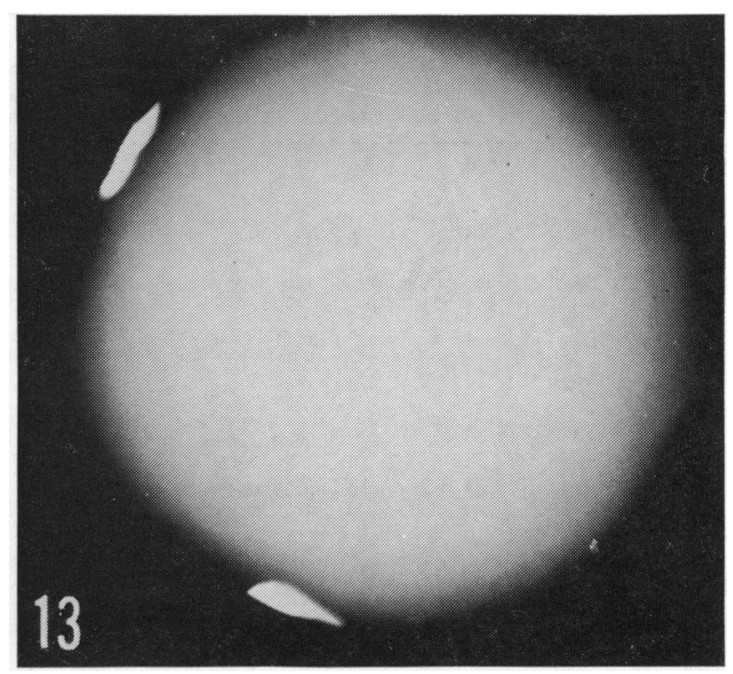

Fig. 12 Intraocular iron wire

Fig. 13 Calcified scleral plaques at sites of insertion of medial and lateral rectus muscles

Fig. 14 Radiodensity in vitreous of glaucomatous eye of patient with sarcoidosis

endophthalmitis, panophthalmitis, segmental retinal gliosis, uveal melanomas, and most eyes with glaucoma.

\section{Discussion}

This study, in which highly sensitive $x$-ray equipment was used, stresses the practical value of roentgenographic examinations in ophthalmic pathology. By means of this technique radiodense foreign bodies can be localised precisely. This greatly facilitates their removal from the eye prior to its processing for microscopic examination. Markedly calcified lesions such as bone, cataracts and scleral plaques, which might create difficulties when tissue 
Table 1 Incidence of calcification in retinoblastomas

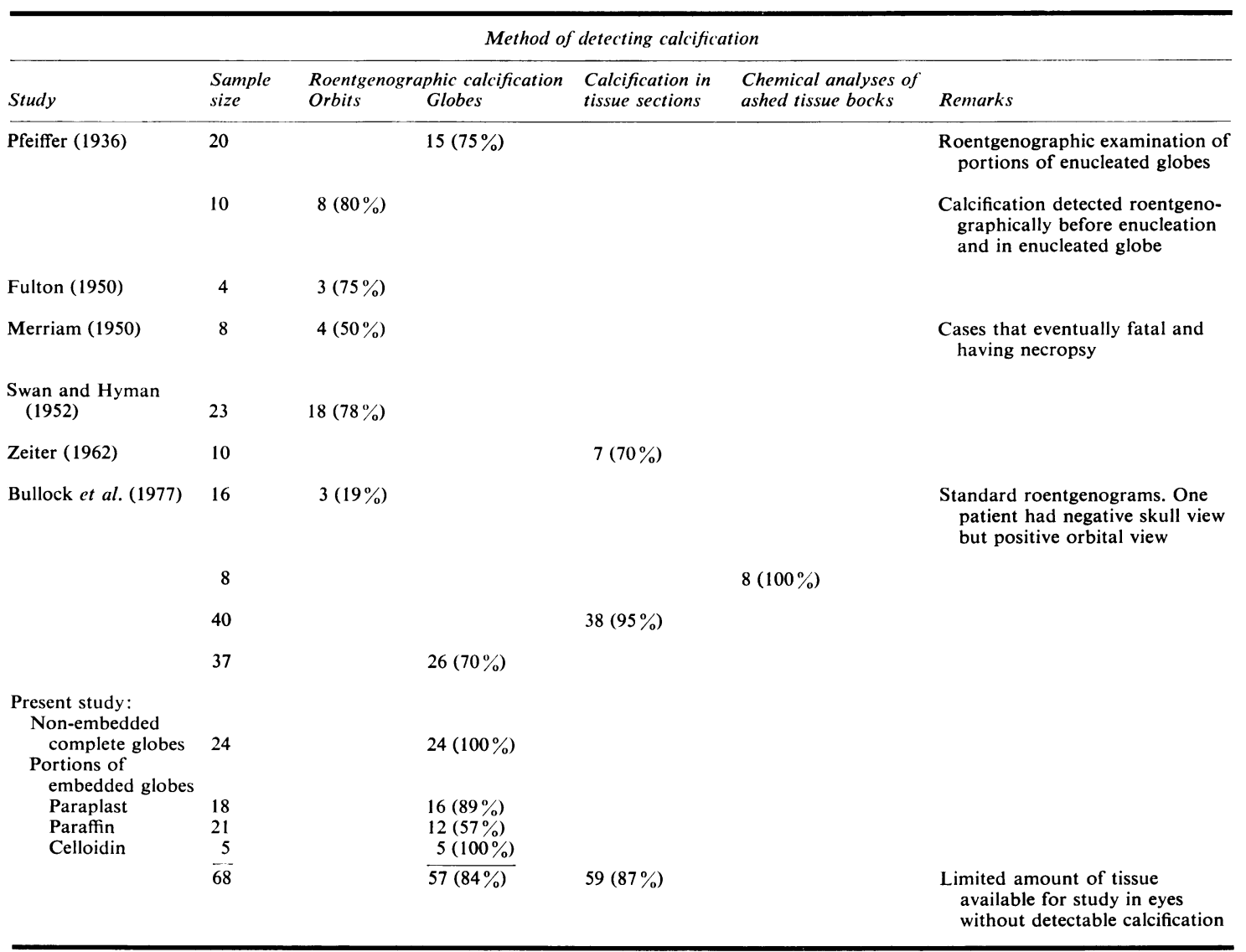

is sectioned, can be detected and decalcified before the eye is embedded. Another virtue of $x$-ray examinations is that radiographs permit the documentation of certain ocular abnormalities better than photographs or tissue sections. For example, some radiopaque materials used in therapeutic procedures dissolve during the routine processing of the tissue.

The tendency of retinoblastomas to calcify, particularly in necrotic portions, has attracted considerable attention. For more than a century calcareous concretions have been recognised in this neoplasm (Knapp, 1869). Depending upon the method of detection, the reported incidence of calcification within retinoblastomas varies considerably (Pfeiffer, 1936; Fulton, 1950; Merriam, 1950; Swan and Hyman, 1952; Zeiter, 1962; Bullock et al., 1977) (Table 1). Bullock et al. (1977) reported radiodensities in only $19 \%$ of cases on standard preoperative roentgenograms, but in ashed tissue blocks with 8 retinoblastomas they found the calcium content in all instances to be much higher than in 4 normal eyes. These investigators found that the quantity of calcium in retinoblastomas with histologically and roentgenographically detectable calcification did not differ from those eyes in which one or both of these techniques failed to detect it. The diagnostic importance of these deposits has been emphasised.

Characteristic white flecks are evident to some degree in virtually all retinoblastomas, and in endophytic and some exophytic neoplasms the areas of calcification are evident ophthalmoscopically. Their clinical appearance is modified by the depth of the pearly or chalk white calcific deposits. Superficial flecks appear discrete, while deeper deposits have more poorly demarcated borders. They are considered to be the most important clinical characteristic of the tumour, and their detection can be decisive in difficult diagnostic cases (Reese, 1976). That retinoblastomas contain areas of calcification of sufficient density and size to be detected by roentgenographic examination is well established (Pfeiffer, 1936; Fulton, 1950; Bullock et al., 1977). In 1936 Pfeiffer drew attention to the practical 
use of this attribute in the clinical evaluation of patients with leucokoria, and stressed the need for roentgenograms of good quality and described technical details of several methods. He recommended stereoscopic films and exposure of the orbit with the patient under general anaesthesia in the Caldwell position as for examination of the sinuses. Other radiographic techniques have been used (Fulton, 1950), but standard radiographs are not reliable in detecting the calcification in retinoblastomas even when it is present (Shields et al., 1976; Bullock et al., 1977). Roentgenographic examinations in young children pose difficulties, and, as a constant success is not obtained, many ophthalmologists unfortunately no longer consider it a useful diagnostic aid.

The high frequency of the radiodensities in retinoblastomas detected in the present study underscores the fact that the vast majority of retinoblastomas not only calcify but have a characteristic radiographic pattern. When present the highly characteristic roentgenograms of retinoblastomas permit the diagnosis to be confirmed with apparent certainty within a few minutes of enucleation without even cutting the globe. The possibility of other calcified lesions being confused with retinoblastomas needs to be considered. Fortunately, most intraocular calcific deposits occur at a different age to retinoblastoma and do not cause confusion clinically. The multiple stippled radiodensities that typify retinoblastomas have not been observed in other entities. In the present study all eyes which were erronously enucleated for suspected retinoblastoma lacked detectable calcification. Other investigators have also not found radiodensities early in life in eyes with lesions that are commonly confused with retinoblastoma (Pfeiffer, 1936; Bullock et al., 1977). However, spotty calcification has been observed in tissue sections of eyes with larval granulomatosis (Howard and Ellsworth, 1965).

Massive gliosis of the retina is unlikely to be confused with retinoblastoma clinically, as it rarely occurs in the same age group. Also, when it is accompanied by calcification, foci of ossification usually coexist (Yanoff et al., 1971). The retinal astrocytic hamartomas of tuberous sclerosis can contain numerous calcospherites, but these too are not abundant early in life. Radiographs of eyes with the aforementioned conditions have not been studied, but because of their histopathological features one is led to suspect that the pattern of radiodensities would differ from that of retinoblastomas. Time will tell whether the characteristic radiographic pattern of retinoblastomas is or is not distinctive for this condition. With retrolental fibroplasia, which has been mistaken clinically for retinoblastoma, the calcification does not occur early in life and is a late manifestation and an expression of ossification in phthisis bulbi. Tayebi (1956) observed roentgenographic evidence of calcification in 14 of 22 eyes with retrolental fibroplasia in children over the age of 4 . It was not found in children under 4 years of age. The present study included phthisical eyes with retrolental fibroplasia from 2 individuals over the age of 20 . Both had radiographic and histological evidence of calcified cataracts and intraocular bone. In neither case did the radiograph resemble that found in retinoblastomas.

In the present study characteristic radiographs were obtained with all 24 retinoblastomas in which the entire eye was $x$-rayed. Negative findings with this tumour were only obtained with $x$-ray examinations performed on portions of eyes. As the calcifications in some retinoblastomas is not uniformly distributed throughout the tumour mass, many of these eyes would probably have contained it if more tissue had been studied. Nevertheless, the possibility that some retinoblastomas may not be detectable by techniques that demonstrate calcification seems highly likely. Experience with portions of embedded globes with retinoblastomas that lacked radiodensities suggests that an absence of radiographically detectable calcium may occur with retinoblastomas that are small or markedly anaplastic. The absence of radiographically detectable calcium clearly is not an indicator of prognosis in individual cases. Calcification was absent in portions of highly invasive anaplastic retinoblastomas as well as in small localised well differentiated tumours that were cured by enucleation. It was likewise also present in retinoblastomas with favourable or unfavourable outcomes.

The clinical diagnosis of retinoblastoma has improved considerably since this potentially lethal intraocular neoplasm first became recognised. Nevertheless, eyes enucleated with suspected retinoblastomas frequently do not harbour a malignancy (Howard, 1969; Kogan and Boniuk, 1962), and even today such errors still occur. Refinements in the clinical distinction between retinoblastomas and other binding conditions of infancy and childhood is clearly of more than academic interest. Unnecessary enucleations during this critical period of orbital development need to be minimised.

Since calcification is so common in retinoblastomas, wider use of $x$-ray examinations and other techniques for the demonstration of calcific deposits in the clinical diagnosis of retinoblastomas is clearly indicated. The development of refined techniques for the clinical detection of ocular calcification 
should be strongly encouraged. Ultrasonography has proved useful in this regard (Sterns et al., 1974; Shields et al., 1976). Shields et al. (1976) have stressed the value of contact B-scan ultrasonography in the diagnosis of retinoblastomas in children whose eyes have opaque media or unusual fundus lesions. Also computerised transaxial tomography, which is intrinsically sensitive to large differences in radiographic density even when the structure is small, can detect intraocular calcification and has been successfully used in the diagnosis of retinoblastomas (Trokel, 1977). In addition the radionuclide technetium diphosphonate, which has an affinity for hydroxyapatite, is being investigated as a possible aid in the diagnosis of retinoblastomas (Bullock et al., 1977). In children some of these modern diagnostic techniques for the detection of calcific deposits unfortunately require general anaesthesia, but the risk of anaesthesia is small and seems justified. Intraocular examinations under anaesthesia have been customary practice for suspected retinoblastomas for a long time.

This study was supported in part by Grants 1R01EY00146 and 5R01-CA13603 from the US Public Health Service.

\section{References}

Bullock, J. D., Campbell, R. J., and Waller, R. R. (1977). Calcification in retinoblastoma. Investigative Ophthalmology and Visual Science, 16, 252-255.

Fulton, H. (1950). A roentgenographic aid in the diagnosis of retinoblastoma. American Journal of Roentgenology and Radium Therapy, 64, 735-739.

Guillot, P., Saraux, H., and Sedan, R. (1966). L'exploration Neuroradiologique en Ophtalmologie. Masson: Paris.
Hartmann, E., and Gilles, E. (1959). Roentgenologic Diagnosis in Ophthalmology. Lippincott: Philadelphia.

Howard, G. M. (1969). Erroneous clinical diagnoses of retinoblastoma and uveal melanoma. Transactions of American Academy of Ophthalmology and Otolaryngology, 73, 199-203.

Howard, G. M., and Ellsworth, R. M. (1965). Differential

diagnosis of retinoblastoma. A statistical survey of 500 children. I. Relative frequency of lesions which stimulate retinoblastoma. American Journal of Ophthalmology, 60, 610-618.

Knapp, H. (1869). In On Intra-ocular tumors, p. 101. William Wood: Philadelphia.

Kogan, L., and Boniuk, M. (1962). Causes for enucleation in childhood with special reference to pseudogliomas and unsuspected retinoblastomas. International Ophthalmology Clinics, 2, 507-521.

Lombardi, G. (1967). Radiology in Neuro-Ophthalmology. Williams \& Wilkins: Baltimore.

Merriam, G. R., Jr. (1950). Retinoblastoma: analysis of seventeen autopsies. Archives of Ophthalmology, 44, 71108.

Pfeiffer, R. L. (1936). Roentgenographic diagnosis of retinoblastoma. Archives of Ophthalmology, 15, 811-821.

Reese, A. B. (1976). Tumors of the Eye, 3rd edn. Harper \& Row: New York.

Shields, J. A., Leonard, B. C., Michaelson, J. B., and Sarin, L. K. (1976). B-scan ultrasonography in the diagnosis of atypical retinoblastomas. Canadian Journal of Ophthalmology, 11, 42-51.

Sterns, G. K., Coleman, D. J., and Ellsworth, R. M. (1974). The ultrasonographic characteristics of retinoblastoma. American Journal of Ophthalmology, 78, 606-611.

Swan, K. C., and Hyman, S. (1952). Experience with tumors of the retina. Archives of Ophthalmology, 47, 416-424.

Tayebi, H. (1956). Ocular calcification and retrolental fibroplasia. American Journal of Roentgenology Radium Therapy and Nuclear Medicine. 76, 583-593.

Trokel, S. L. (1977). Unpublished observations.

Yanoff, M., Zimmerman, L. E., and Davis, R. L. (1971). Massive gliosis of the retina. International Ophthalmology Clinics, 2, 211-229.

Zeiter, H. J. (1962). Calcification and ossification in ocular tissue. American Journal of Ophthalmology, 53, 265-274. 